\title{
Factors That Impact the Vacuum Ultraviolet Photofragmentation of Peptide Ions
}

\author{
Matthew S. Thompson, Weidong Cui, and James P. Reilly \\ Department of Chemistry, Indiana University, Bloomington, Indiana, USA
}

Several groups have investigated the photodissociation of peptide ions with ultraviolet light. Significant differences have been reported with 157 and $193 \mathrm{~nm}$ excitation. Recent studies have shown that the mass analyzer can also influence the observed photofragment distribution. Comparison of experiments using different peptides, wavelengths, and mass analyzers is undesirably complicated. In the present work, several peptides are analyzed with both 157 and $193 \mathrm{~nm}$ photodissociation in tandem-TOF and linear ion trap mass spectrometers. The results indicate that the fragment ion distribution can be influenced by both the photodissociation wavelength and the mass analyzer. The two wavelengths generate similar spectra in an ion trap but quite different results in a tandem-TOF instrument. (J Am Soc Mass Spectrom 2007, 18, 1439-1452) @ 2007 American Society for Mass Spectrometry

$\mathrm{U}$ ltraviolet laser photodissociation is an appealing method for inducing fragmentation in peptide mass spectrometry experiments. Direct fragmentation is possible since the photon energies are higher than peptide bond strengths, and photodissociation can be coupled to a variety of mass analyzers. Several groups have successfully applied this technique, but the resulting fragmentation patterns exhibit significant variation. Experimental differences complicate comparison between studies and have thus far made it difficult to characterize the photodissociation phenomenon.

Laser photodissociation requires a light source that matches an absorption band of the analyte. Several peptide absorption bands have been observed in the far ultraviolet [1]. Experimental and theoretical studies have identified one band centered around $200 \mathrm{~nm}$ [2] and a second at $160 \mathrm{~nm}[3,4]$. The former can be excited with an ArF laser at $193 \mathrm{~nm}$ and the latter with an $\mathrm{F}_{2}$ laser at $157 \mathrm{~nm}$. Although different electronic states are involved, both transitions have been associated with the backbone amide [1-4]. One hundred ninety-three $\mathrm{nm}$ light has some practical advantages compared with 157 nm light, and has been more widely used. High power $193 \mathrm{~nm}$ lasers are available, and the light is compatible with fused silica optics. One hundred fifty-seven $\mathrm{nm}$ light has a higher photon energy, but requires exotic optics and cannot be transmitted through air.

The most commonly used activation technique for tandem mass spectrometry of peptides is collision induced dissociation (CID). Instruments that use this method include the ion trap, triple quadrupole, and quadrupole-TOF. Activation occurs via a large number

Address reprint requests to Dr. J. P. Reilly, Department of Chemistry, Indiana University, Bloomington, IN 47405-4001, USA. E-mail: reilly@ indiana.edu of low-energy collisions with gas molecules that gradually increase the total internal energy of the analyte ion until it undergoes decomposition. The resulting fragments are typically $b$ and $y$ ions, which are referred to as "low-energy" fragments. In contrast, "high-energy" excitation techniques induce fragmentation as a result of a single, highly energetic activation event, typically a collision with gas or a surface. The most widely used high-energy technique is high-energy CID in a MALDI tandem-TOF instrument, in which a single collision event at 1 to $2 \mathrm{keV}$ energy induces fragmentation. Inefficient conversion of collision energy into internal energy causes the total energy imparted by this technique to be of the same magnitude as the absorption of a VUV photon. Since internal energies above the dissociation threshold can be achieved with these techniques, many additional fragmentation processes become possible. The additional fragment types are referred to as "high-energy" fragments. A review of these techniques is available [5].

Photodissociation of di- and tripeptide ions with 193 $\mathrm{nm}$ light was first reported by Bowers et al. using an FTICR mass analyzer [6]. Larger peptides have also been investigated with this technique [7-9]. Photodissociation at this wavelength was subsequently studied in a four-sector mass spectrometer [10]. In each case, a range of fragment ions was generated, including those commonly observed from vibrationally excited ions and those associated with higher energy fragmentation processes. When comparable internal energies were deposited with $193 \mathrm{~nm}$ light and surface induced dissociation in an FTICR, the observed fragmentation patterns were quite similar [9].

UV photodissociation is a particularly appealing technique to couple with TOF instruments. The light pulse can be synchronized to the fragment ion of interest, and photodissociation does not affect the per- 
formance of the mass analyzer. One hundred ninetythree $\mathrm{nm}$ photodissociation has been applied in several different MALDI-TOF instruments [11-17]. Early studies [11-15] generated fragmentation patterns consistent with the previously described FTICR [6-9] and sector experiments [10]. We have performed photodissociation experiments in a MALDI tandem-TOF instrument using $193 \mathrm{~nm}$ light [14], and we subsequently employed $157 \mathrm{~nm}$ light for the same purpose [16, 17]. The $193 \mathrm{~nm}$ photofragment distributions obtained on this apparatus were consistent with the studies described above. When $157 \mathrm{~nm}$ photodissociation was applied to arginineterminated peptides, distinctive fragmentation patterns were observed: an extensive series of $x$ ions is observed if an arginine residue is located at the C-terminus, and $a$ ions are observed when arginine is at the $\mathrm{N}$-terminus. These are accompanied by $d, v$ or $w$ ions resulting from side-chain loss, along with limited numbers of lowenergy fragments. A mechanism involving a wavelength-specific photolytic cleavage of the $\alpha$-carbon carbonyl-carbon bond while the charge proton remains sequestered at arginine has been proposed [17]. While both the $a$ - and $x$-type fragments are consistent with this model, the $x$ fragment series is more unusual, as $a$-type ions are frequently observed with other high-energy peptide excitation techniques [18]. Since the clearest evidence of a unique fragmentation mechanism in 157 $\mathrm{nm}$ photodissociation comes from peptides with Cterminal arginine, these peptides should be the focus of comparative studies. In the previously described 193 $\mathrm{nm}$ photodissociation studies, only one peptide with a C-terminal arginine residue was investigated, and only $y$-type fragments were observed [19].

Several recent studies have suggested that the mass analyzer can influence photofragmentation patterns. For example, we have reported $157 \mathrm{~nm}$ photodissociation of singly-charged arginine-containing peptides using a linear ion trap [20]. As with the previously described tandem-TOF photodissociation experiments, a collection of high-energy $a$ or $x$ fragments was observed. However, the abundance of $b$ or $y$ fragments was found to increase significantly in the ion trap. We suggested that the long observation timescale on this instrument makes additional fragments observable [20]. Zubarev and coworkers have also applied this technique to cleave disulfide bonds [21] and investigate peptide zwitterions in the gas-phase [22, 23]. Morgan and Russell have compared $193 \mathrm{~nm}$ photodissociation in a tandem-TOF to their prior work using stepped reflectron voltages, and have studied some peptides with C-terminal arginine. Photodissociation spectra from the tandem-TOF were found to contain fewer $b$ and $y$ fragments [19]. Kim and coworkers have used the same wavelength to fragment several arginine-terminated peptides in a MALDI curved-field reflectron apparatus [24-26]. The resulting fragmentation patterns exhibit significant similarity with our $157 \mathrm{~nm}$ tandem-TOF photodissociation results. They have recently developed a
MALDI tandem-TOF instrument and performed $193 \mathrm{~nm}$ photodissociation of several large peptides [27].

The fragmentation patterns reported in these peptide photodissociation studies vary significantly, suggesting that both the mass analyzer and wavelength influence the observed spectra. To evaluate the influence of each of these experimental parameters requires the generation of appropriate data. In the present work 193 and $157 \mathrm{~nm}$ light are used to photodissociate a collection of peptides in both tandem-TOF and linear ion trap mass spectrometers. Three of the selected peptides have also been studied by Kim and coworkers using $193 \mathrm{~nm}$ photodissociation in their curved-field reflectron instrument. A variety of comparisons can be made, and the effect of each parameter can be separately evaluated.

\section{Experimental}

\section{Sample preparation}

Glu-fibrinopeptide B, FSWGAEGQR and Substance P were purchased from Sigma (St. Louis, MO). Peptide AAAANSAAAR was custom synthesized by Thermo Electron (Waltham, MA). ALELFR was obtained from trypsin digestion of horse myoglobin (Sigma) and purified via reserve phase HPLC. CHCA matrix was obtained from Aldrich (St. Louis, MO) and used without purification. For MALDI experiments, $10 \mathrm{~g} / \mathrm{L}$ CHCA was dissolved in 1:1 acetonitrile:water containing $0.1 \%$ trifluoroacetic acid. Peptide and matrix were mixed 1:9 and $1 \mu \mathrm{L}$ samples were deposited on the probe. Each spot contained $10 \mathrm{pmol}$ of peptide.

\section{Photodissociation}

The homebuilt tandem time-of-flight apparatus used for photodissociation experiments has been described previously [17]. It is similar to tandem time-of-flight mass spectrometers that employ collision cells $[28,29]$ except that ion fragmentation is induced by a pulse of UV light. Briefly, MALDI-generated ions are separated in the first flight tube. Post-source decay fragments are also formed at this time, and these fragments travel with precursor ions to the source of the second stage. The laser beam intersects the ion packet just before the second stage source. The precursor and all PSD and photofragments are then re-accelerated and separated in the second mass analyzer. 193 and $157 \mathrm{~nm}$ photodissociation light is generated by an excimer laser (Lambda Physik LPF205; Orlando, FL). The laser light is introduced into the mass spectrometer via a fused silica window for $193 \mathrm{~nm}$ experiments. $157 \mathrm{~nm}$ light is absorbed by air, so the light path is evacuated. For routine experiments, $\sim 1 \mathrm{~mJ}$ of $157 \mathrm{~nm}$ light is directed through a $5 \mathrm{~mm} \times 10 \mathrm{~mm}$ aperture; $193 \mathrm{~nm}$ light from $\mathrm{ArF}$ is also generated in the same laser. Larger pulse energies are available at this wavelength, but $2.5 \mathrm{~mJ}$ is typically used. For energy-dependence experiments, the laser is attenuated using reflection and absorption by 


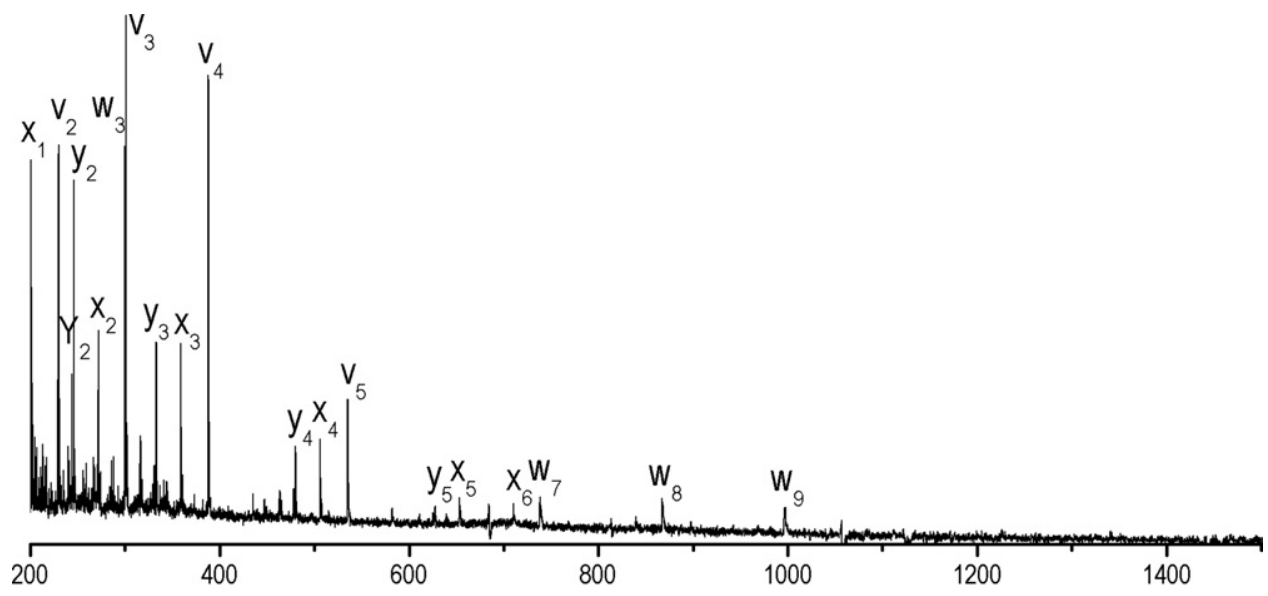

(a)

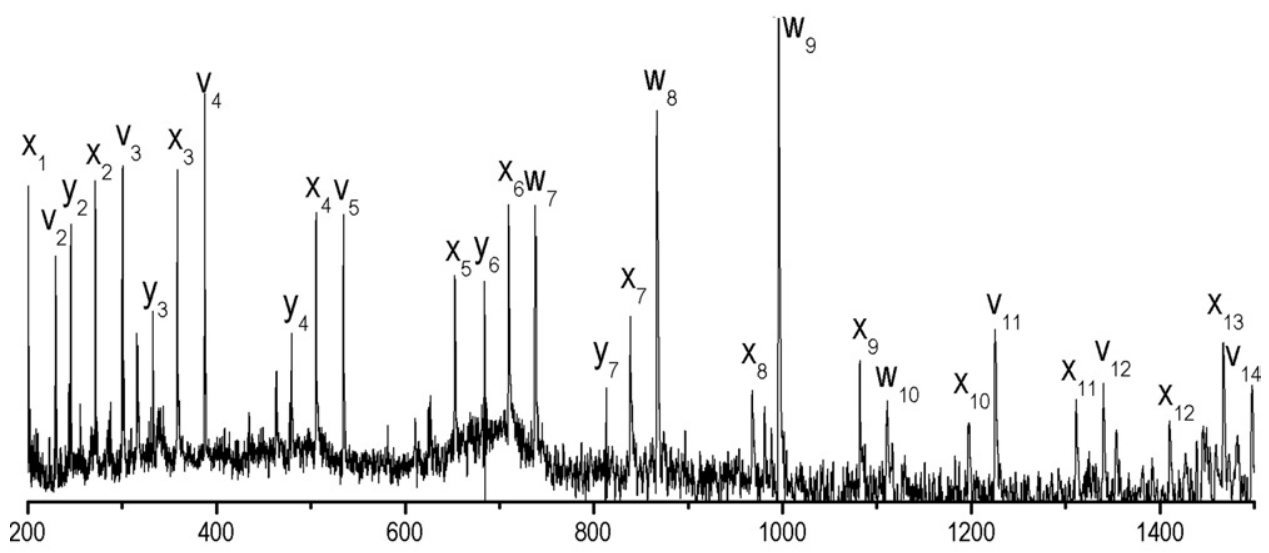

(b)

Figure 1. Tandem-TOF photodissociation of Glu-fibrinopeptide B (EGVNDNEEGFFSAR) using (a) 193 and (b) $157 \mathrm{~nm}$ light.

sapphire plates, and pulse energies are measured with an Ophir (Wilmington, MA) Nova II joule meter equipped with a PE50 pyroelectric head.

Post-source decay fragments are formed in all experiments in this apparatus, and they complicate the interpretation of photofragmentation patterns. To make it possible to distinguish post-source decay and photodissociation fragments, the photodissociation laser is fired on alternate MALDI laser shots and the data are averaged separately. Because these spectra are recorded quasi-simultaneously, the post-source decay contribution is similar. The spectrum recorded with the photodissociation laser off is subtracted from the laser-on spectrum to obtain the photodissociation only spectra presented here.

The apparatus used for linear ion trap experiments has been described previously [20]. Briefly, a $3 \mathrm{uM}$ solution of the peptide is prepared in a solvent of 1:1 acetonitrile:water containing $1 \%$ acetic acid. The peptide of interest is electrosprayed via direct infusion into a ThermoFinnigan LTQ linear ion trap (Waltham, MA). The instrument is configured to acquire a tandem mass spectrum of the singly-charged peptide with collision energy set to $0 \%$ and $\mathrm{Q}$ of 0.1 to reduce the low-mass cutoff. During the activation period, a pulse of photodissociation light at either 193 or $157 \mathrm{~nm}$ generated by a GAM EX100HF (Orlando, FL) is introduced through a $1.7 \mathrm{~mm}$ aperture aligned with the back aperture of the ion trap. A separate spectrum is acquired of blank electrospray solvent and used to perform background subtraction of photoion signal.

\section{Results}

Tandem photodissociation mass spectra of Glu-fibrinopeptide B (EGVNDNEEGFFSAR) were obtained using 193 and $157 \mathrm{~nm}$ light in both the tandem-TOF and linear ion trap. The $193 \mathrm{~nm}$ photodissociation spectrum (Figure 1a) contains an $x$ ion series extending from $x_{1}$ to $x_{6}$, as well as a series of $y$ ions containing $y_{2}$ through $y_{5}$. No fragments larger than $w_{9}$ are observed. The $157 \mathrm{~nm}$ photodissociation spectrum (Figure $1 \mathrm{~b}$ ) contains a more extensive collection of fragment ions, particularly in the range above $m / z 1000$. A complete $x$ ion series extending from $x_{1}$ to $x_{13}$ is observed for this 14 residue peptide, and many of these $x$ ions are accompanied by $v$ or $w$ 


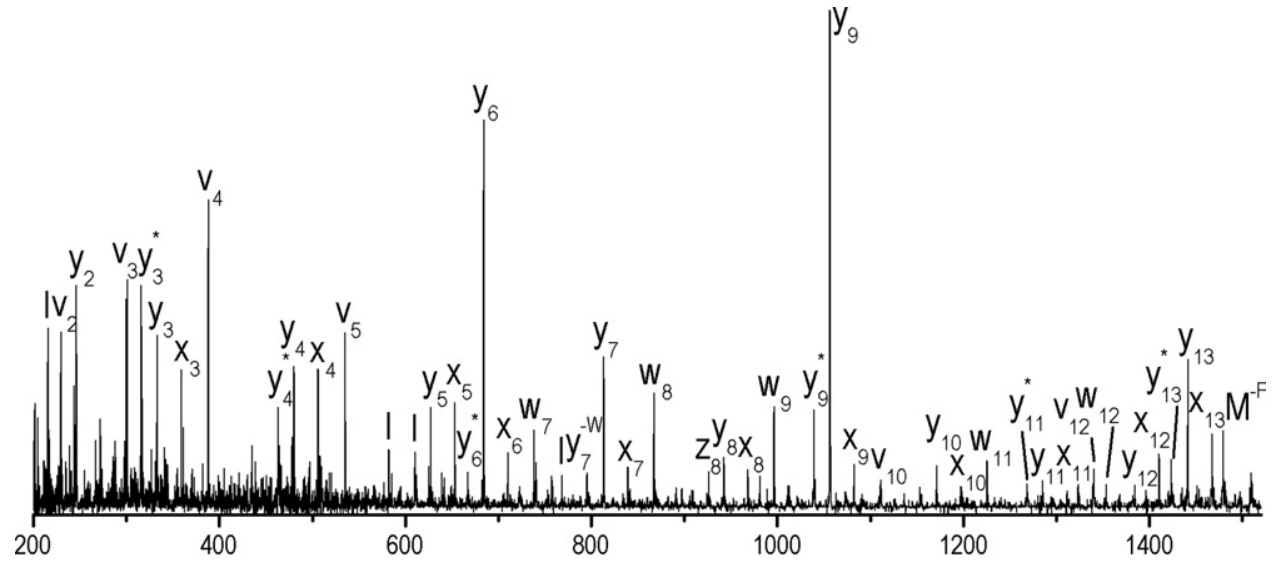

(a)

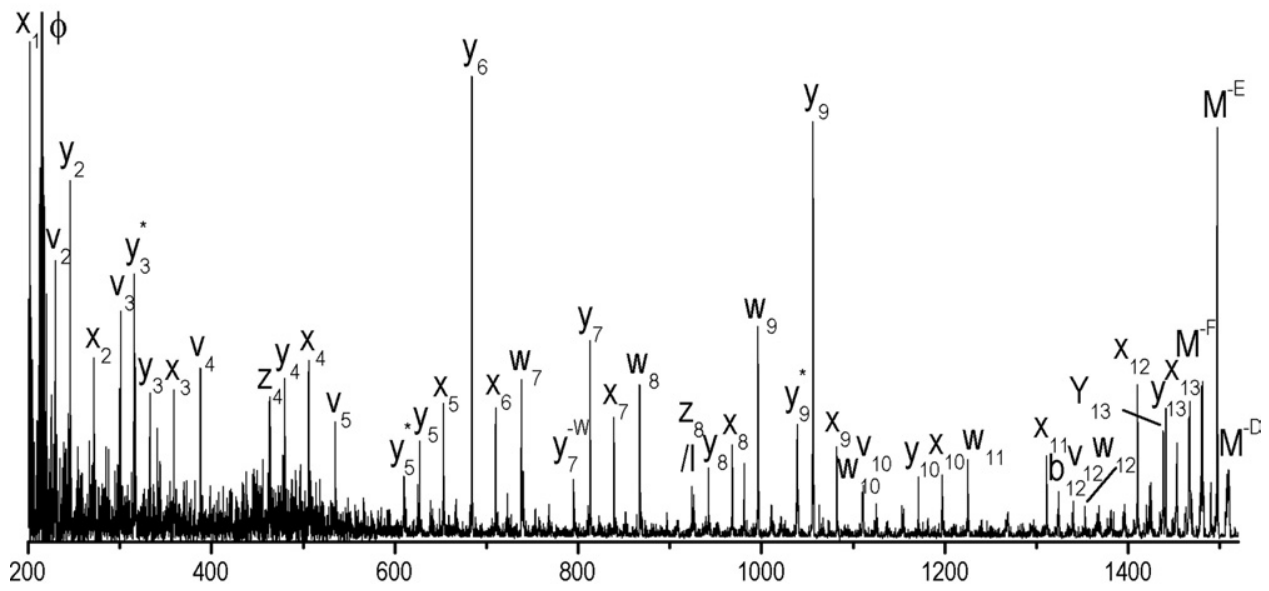

(b)

Figure 2. Linear ion trap photodissociation of Glu-fibrinopeptide B (EGVNDNEEGFFSAR) using (a) 193 and (b) $157 \mathrm{~nm}$ light. Peaks labeled phi $(\phi)$ are photoions not fully removed during background subtraction. An asterisk $(*)$ indicates loss of $\mathrm{NH}_{3}$.

fragments. Five $y$ fragments: $y_{2}, y_{3}, y_{4}, y_{6}$ and $y_{7}$ are observed. There are significant differences between the two wavelengths in the high-mass region, but a few similar features are observed. $w_{8}$ and $w_{9}$ are the two most intense peaks in the $157 \mathrm{~nm}$ spectrum, and they are the only fragments observed above $\mathrm{m} / \mathrm{z} 600$ in the $193 \mathrm{~nm}$ spectrum.

In contrast with the significant differences observed between the two photodissociation wavelengths in the tandem-TOF, the photodissociation spectra of Glufibrinopeptide $\mathrm{B}$ obtained in the linear ion trap are strikingly similar. The $193 \mathrm{~nm}$ spectrum (Figure 2a) contains a collection of fragment ions across the mass range that includes $11 x$-type ions. The $157 \mathrm{~nm}$ ion trap spectrum (Figure $2 b$ ) contains a complete series of $14 x$ ions. Similar extensive collections of $y$ fragments are present in both spectra: 11 with $193 \mathrm{~nm}$ and 10 with 157 $\mathrm{nm}$ photodissociation. Two of these ions, $y_{6}$ and $y_{9}$ are the strongest peaks in both ion trap spectra, while $y_{6}$ is weak and $y_{9}$ is not observed at either wavelength in the TOF instrument. These two $y$ ions are associated with the well-known preferential cleavage C-terminal to an acidic amino acid [30]. Several y fragments are accompanied by $y^{*}$ ions, with the ${ }^{*}$ representing loss of $\mathrm{NH}_{3}$. $y_{13}$ is accompanied by a peak $2 \mathrm{Da}$ lighter, labeled $Y_{13}$. The lower case $y$ label indicates the common $y$ ion, resulting from cleavage of the peptide bond with $2 \mathrm{H}$ atoms transferred to the resulting fragment. The lighter capital $Y$ fragments result from the same backbone cleavage, but are $2 \mathrm{Da}$ lighter because no $\mathrm{H}$ transfer occurs [31]. Occasionally, photoion peaks are not completely removed from ion trap photodissociation spectra despite background subtraction of a photoion-only spectrum. One such peak is present in Figure 2b at 215 $\mathrm{m} / \mathrm{z}$ and is labeled $\phi$ (phi).

Photodissociation spectra from the tandem-TOF instrument of another C-terminal arginine containing peptide AAAANSAAAR are displayed in Figure 3. With $193 \mathrm{~nm}$ light (Figure 3a), the $x$ ion series obtained from this peptide extends only from $x_{1}$ to $x_{4}$. A number of $v$ and $w$ ions are observed, but the heaviest interpretable fragment is $w_{6}$. Several $y$ - and $b$-type ions also appear. In contrast, the $157 \mathrm{~nm}$ tandem-TOF spectrum of this peptide (Figure $3 \mathrm{~b}$ ) contains a complete $x$ ion 


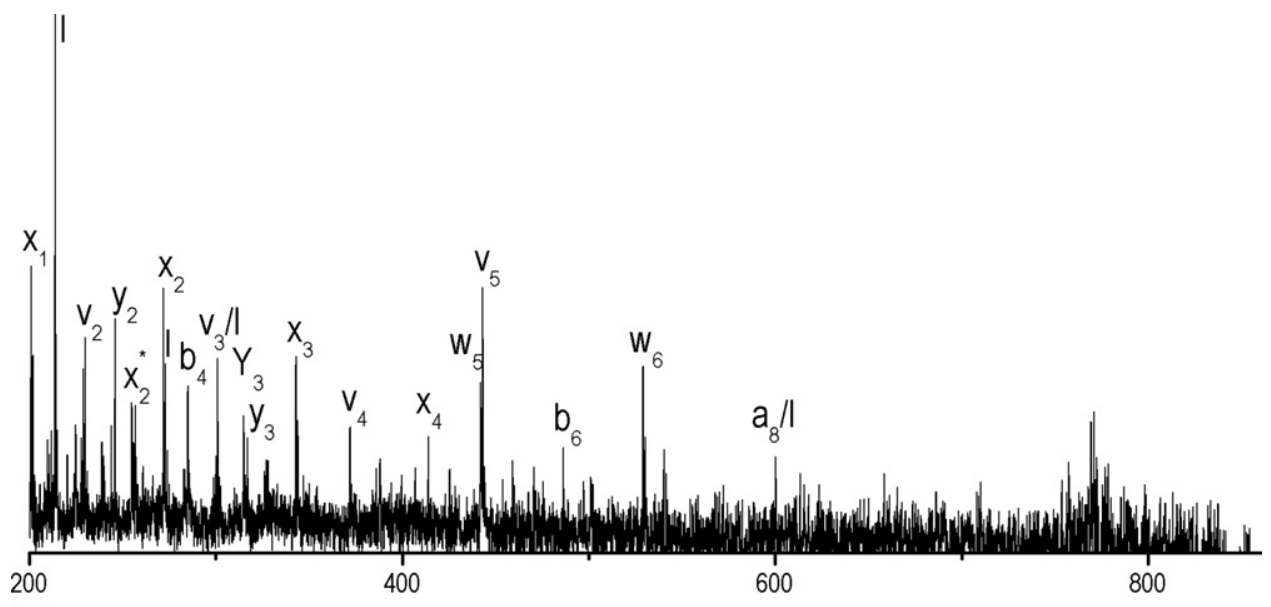

(a)

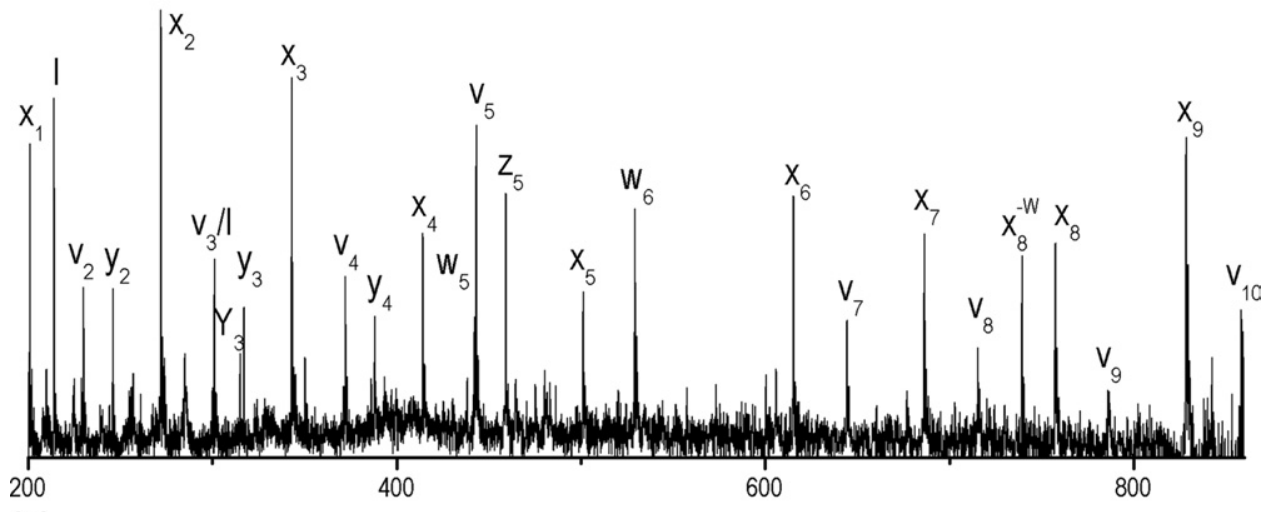

(b)

Figure 3. Tandem-TOF photodissociation of the synthetic peptide AAAANSAAAR using (a) 193 and (b) $157 \mathrm{~nm}$ light.

series from $x_{1}$ to $x_{9}$ accompanied by significant $v$ and $w$ fragment series. N-terminal fragments are either absent or of low intensity. Photodissociation of this peptide in the linear ion trap yields considerably more complex spectra. With $193 \mathrm{~nm}$ light (Figure 4a), major peaks include $x_{9}, v_{10}, b_{7}{ }^{*}$ and $b_{8}^{*}$. An $x$ ion series missing only $x_{5}$ is observed. Several other fragment series have similar coverage, including a complete $y$ fragment series, as well as a number of $Y$ ions. Remarkably, several $\mathrm{N}$-terminal fragments are also present, with $7 b$ and $4 a$ ions observed. Two particularly intense peaks occur at adjacent masses of 828 and $829 \mathrm{Da}$. The former is assigned as an $x_{9}$ fragment while the latter is interpreted as M-44 (loss of $\mathrm{CO}_{2}$ from the precursor ion). The 828 peak may have a small contribution from M-45 (loss of $\mathrm{COOH}$ from the precursor). Zubarev and coworkers have interpreted M-44 and M-45 fragments as indicative of whether the C-terminus is deprotonated by a zwitterionic structure [23]. While many of the same high-energy fragments are present when the peptide is dissociated with $157 \mathrm{~nm}$ light in the ion trap (Figure 4b), the $x_{9}$ and $x_{8}$ peaks are particularly dominant. Many of the low-energy fragments observed with $193 \mathrm{~nm}$ light are also present, but the intensity and number of
N-terminal low-energy fragments are lower with 157 nm light than with $193 \mathrm{~nm}$ light.

Tandem-TOF photodissociation spectra of a third C-terminal arginine peptide FSWGAEGQR are shown in Figure 5. With $193 \mathrm{~nm}$ light (Figure 5a), this peptide yields a more extensive collection of fragments than the previous examples. A nearly complete $x$ ion series is observed, with only $x_{7}$ missing, as well as several $v$ ions and $5 y$ ions. The peak intensities do not exhibit the previously observed mass dependence. Another unusual feature of this spectrum is the peak labeled M $\mathrm{W}$, which represents the loss of a tryptophan side chain from the precursor. Only minor differences are observed when this molecule is fragmented with $157 \mathrm{~nm}$ light (Figure $5 \mathrm{~b}$ ). The previously missing $x_{7}$ ion appears, making the series complete, and the relative intensities of all $x$ ions are higher than with $193 \mathrm{~nm}$ light. The $y$ fragment series is similar with both wavelengths, and two additional side-chain losses are observed with 157 $\mathrm{nm}$ photodissociation.

Photodissociation spectra of FSWGAEGQR acquired in the linear ion trap are displayed in Figure 6. Both 193 (Figure 6a) and $157 \mathrm{~nm}$ (Figure 6b) photodissociation generate a complete series of $x$ ions. Many of the ions 


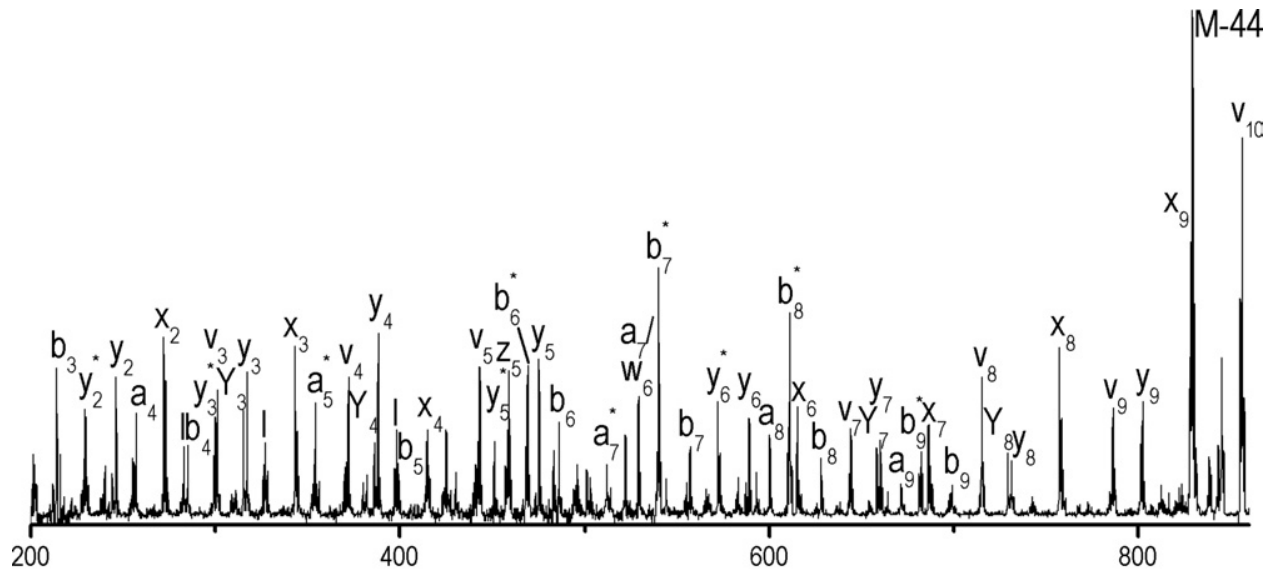

(a)

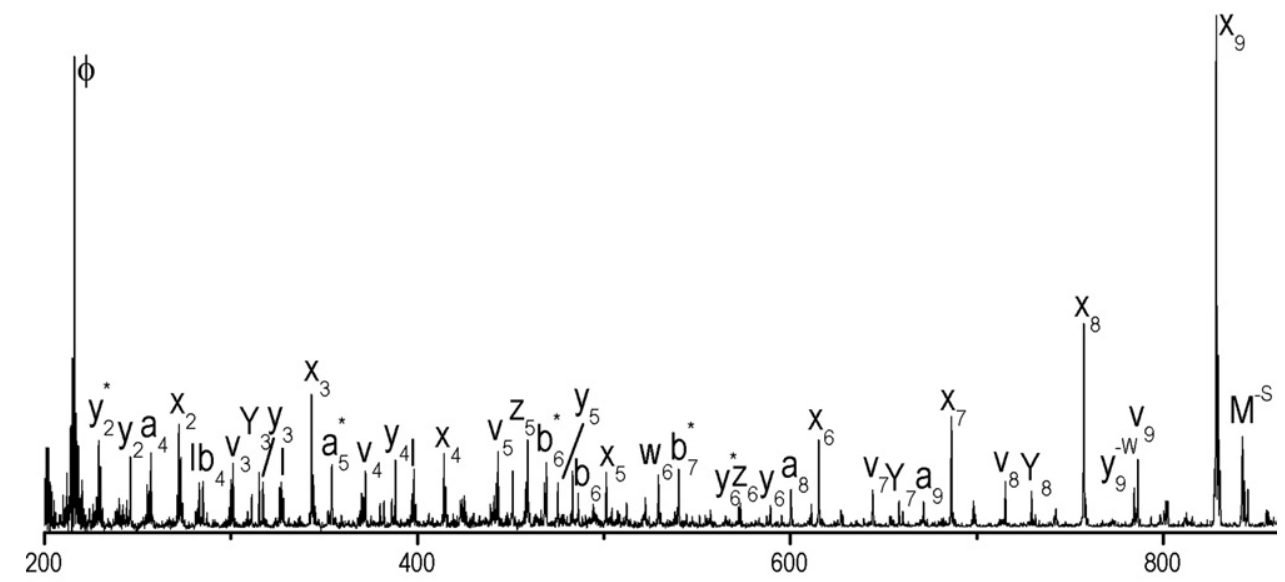

(b)

Figure 4. Linear ion trap photodissociation of the synthetic peptide AAAANSAAAR using (a) 193 and (b) $157 \mathrm{~nm}$ light.

observed in the tandem-TOF spectra are present, but several additional fragments are observed in both, including $2 b$ and $2 z$ fragments that were not present in either tandem-TOF spectrum. The $y_{3}$ peak, corresponding to cleavage $\mathrm{C}$-terminal to glutamic acid, is intense in both ion trap spectra.

The $193 \mathrm{~nm}$ tandem-TOF photodissociation spectrum of Substance P (RPKPQQFFGLM-NH ${ }_{2}$ ) is shown in Figure 7a. Consistent with prior experiments using $\mathrm{N}$-terminal arginine peptides, primarily $a$ - and $d$ - type fragments are observed. A nearly complete collection of $a$ - type fragments extending to $a_{8}$ is generated, although the intensity of the fragments decreases monotonically with mass. When $157 \mathrm{~nm}$ light is used to induce fragmentation (Figure $7 \mathrm{~b}$ ), many of the same fragments are observed, but the high-mass fragments are significantly more intense, including two high mass fragments $\left(a_{10}\right.$ and $\left.d_{11}\right)$ that were not observed at $193 \mathrm{~nm}$. The $a_{9}$ fragment associated with cleavage C-terminal to a glycine residue is missing with both wavelengths. In both spectra, a few other N-terminal fragments are observed.

As with the other peptides studied, the photodissociation spectra of Substance P obtained in the linear ion trap contain a number of additional fragment types that were not observed with the tandem-TOF. The $193 \mathrm{~nm}$ ion trap spectrum (Figure 8a) contains an $a$ ion series extending from $a_{3}$ to $a_{8}$ as well as $4 b$-type fragments. There are $3 c$ and $2 y$ fragments, as well as many corresponding to neutral loss of $\mathrm{NH}_{3}$ from these ions. The overall distribution with $157 \mathrm{~nm}$ light (Figure 8b) is quite similar, although one $x$ ion $\left(x_{9}\right)$ is also observed. When compared with the tandem-TOF spectra, the ion trap experiments contain significant additional lowenergy fragment signal.

To investigate whether multiphoton processes might be influencing the fragmentation processes that we observe, the incident laser power at both light wavelengths was varied for both instruments. The tandemTOF results are of particular interest, since significant wavelength dependence was observed on this instrument and because the loss of high-mass fragment ions in Figures 1a, 3a, and 5a might be interpretable as an intensity-dependent effect. $193 \mathrm{~nm}$ photodissociation of ALELFR is shown at three laser pulse energies in Figure 9. At the lowest energy (Figure 9a), a poor signal to noise ratio is obtained, and large fragment ions are 


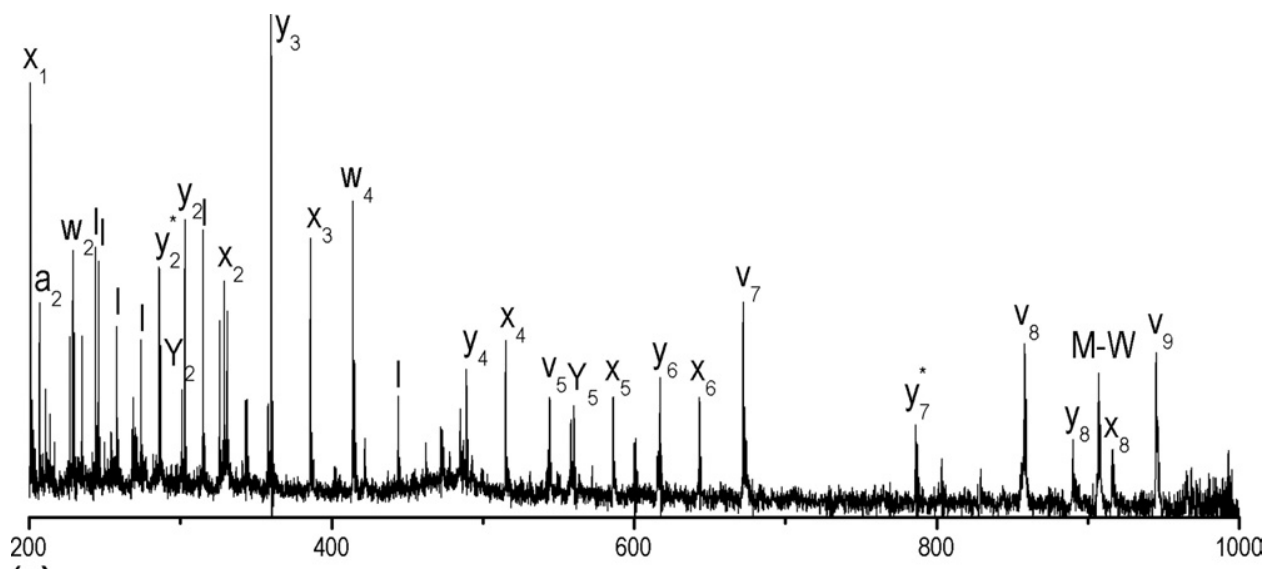

(a)

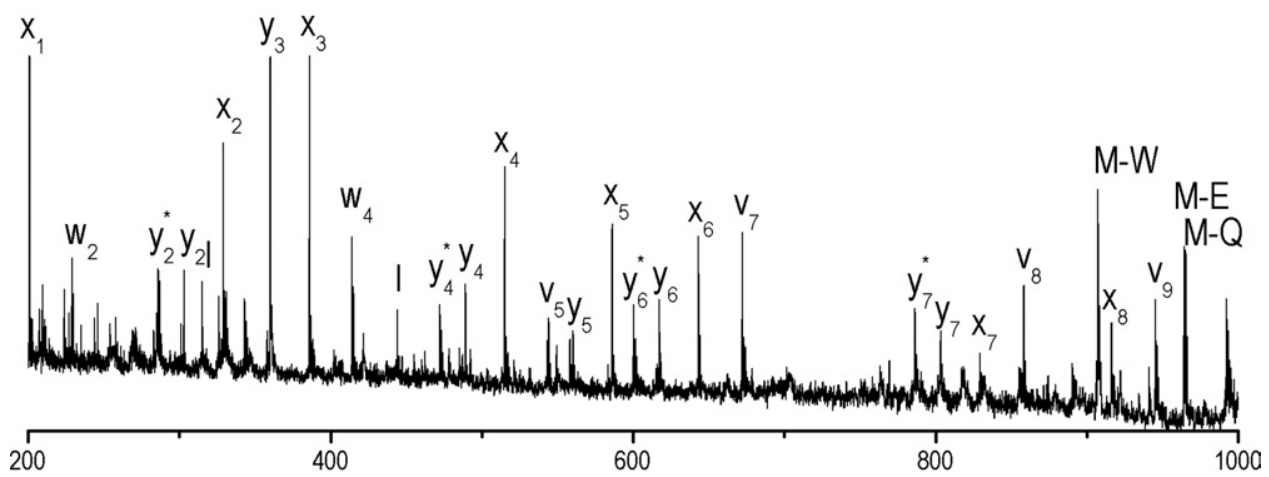

(b)

Figure 5. Tandem-TOF photodissociation of FSWGAEGQR using (a) 193 and (b) 157 nm light. Peaks labeled $\mathrm{M}-X$ represent loss of an amino acid side-chain from the precursor.

missing. As the laser energy increases (Figure $9 \mathrm{~b}$ and $\mathrm{c}$ ), there is an overall signal to noise improvement, and there are small changes in the relative intensities of peaks, but the spectrum does not change dramatically. With $157 \mathrm{~nm}$ light (Figure 10) the intensity could only be varied by a factor of four. At the lowest laser power (Figure 10a), the spectrum is quite noisy, but a complete series of $x$ ions is observed. As the power is increased, the overall signal to noise ratio of the spectrum improves, but the distribution of large fragment ions does not change significantly. The abundance of low-mass immonium ions appears to increase at higher laser energies.

\section{Discussion}

The preceding results, summarized in Table 1, demonstrate that both the wavelength and mass analyzer play a significant role in determining the fragments that are observed in peptide photodissociation spectra. To compare experiments performed at different wavelengths, several factors must be considered. As previously described, 157 and $193 \mathrm{~nm}$ light excite different electronic states that are associated with peptide backbone amides. The differences observed between the two wavelengths in the tandem-TOF experiments could be caused by either distinct photolysis processes associated with the two electronic states or by differences in the total energy imparted to the analyte ion (6.4 and 7.8 $\mathrm{eV}$ per photon for 193 and $157 \mathrm{~nm}$ light). To evaluate these possibilities, the number of photons absorbed by the peptide must be known, and must be consistent between all experiments. The number of $193 \mathrm{~nm}$ photons required to fragment a peptide precursor ion has been investigated by Gimon-Kinsel et al. [32]. By measuring precursor depletion as a function of laser power they showed that photodissociation occurs via a singlephoton process. Since the photon energy of $157 \mathrm{~nm}$ light is higher than that of $193 \mathrm{~nm}$ light, photodissociation at the former wavelength certainly could be a singlephoton process. However, the mass-dependent fragment ion distributions described above for several 193 $\mathrm{nm}$ in the tandem-TOF photodissociation experiments follow a trend that could be explained by the absorption of additional photons by precursor or fragment ions [33].

To resolve this uncertainty, we have evaluated the laser fluence dependence of our mass spectra. For the molecules discussed here and for several others, we performed experiments in which the laser intensity was varied using both wavelengths and both mass spectrometers. The results from ALELFR in the tandem-TOF 


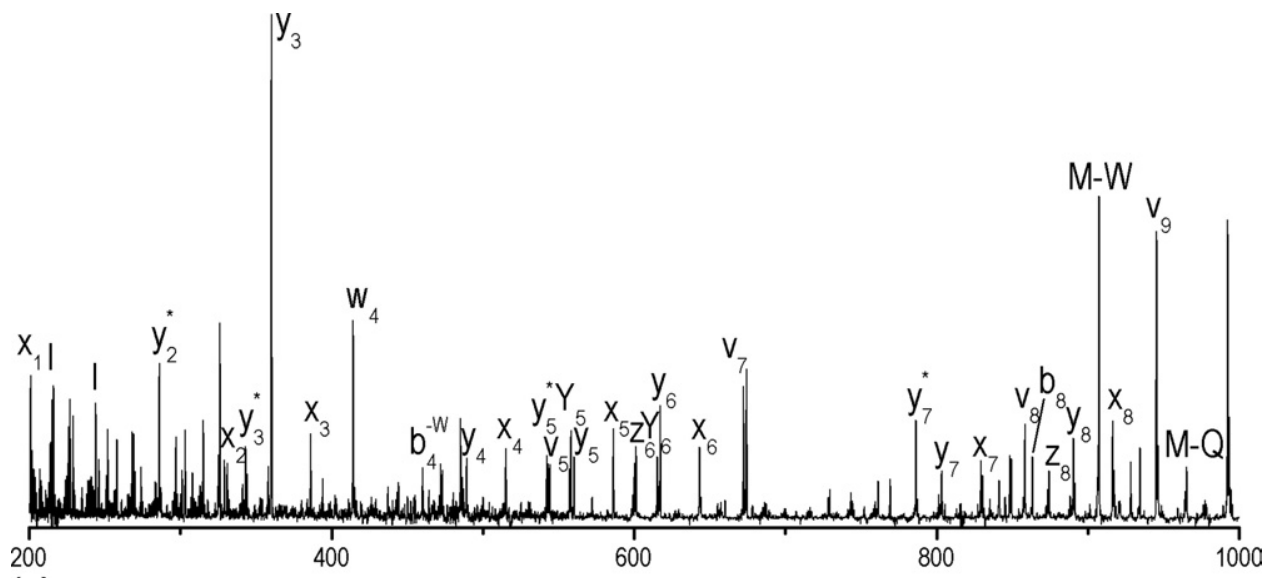

(a)

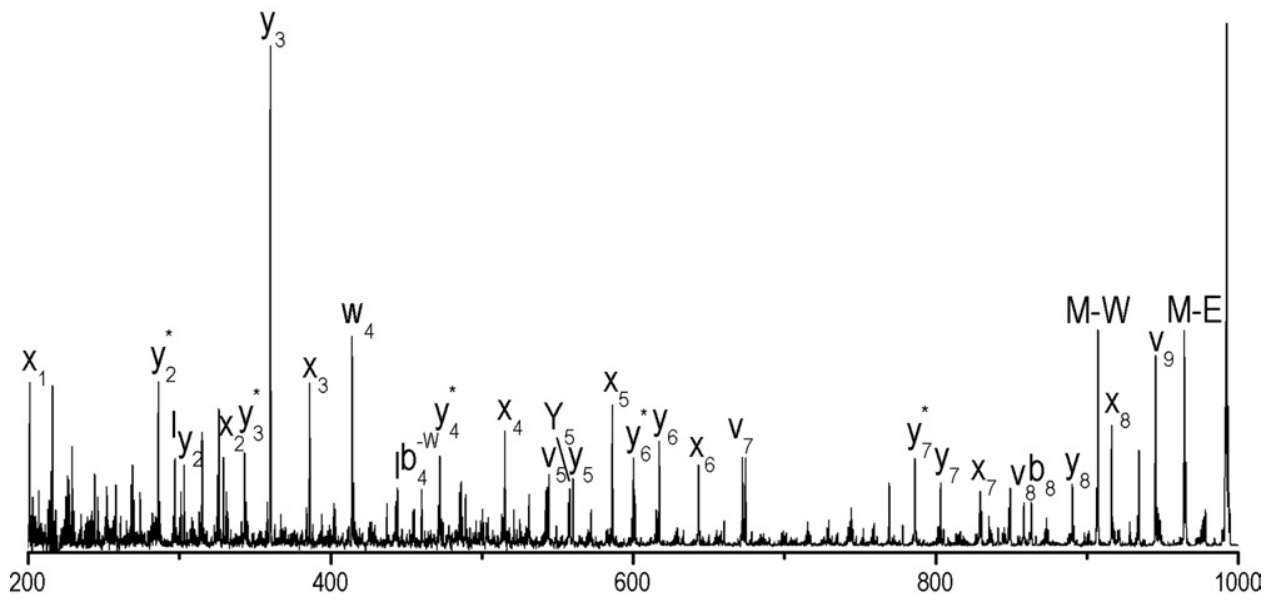

(b)

Figure 6. Linear ion trap photodissociation of FSWGAEGQR using (a) 193 and (b) $157 \mathrm{~nm}$ light.

are shown in Figures 9 and 10. While a bias favoring small fragments was observed in most of our $193 \mathrm{~nm}$ TOF spectra, the fragment ion distributions were relatively independent of light intensity. The fragment ion signal increased with laser power, but the only change in the overall distribution was a small increase in the abundance of low-mass fragment ions with high laser powers at both wavelengths. We found that reducing the photodissociation laser intensity below the levels used for these experiments yielded a reduction in the abundance of all fragment ions without any significant change in the fragment distribution. We could not find conditions where high-mass fragments were observed at this wavelength in the tandem-TOF. One hundred ninety-three $\mathrm{nm}$ photodissociation of FSWGAEGQR appears to be an exception to these trends, and will be discussed below. The lack of significant changes in the distribution of high-mass fragments as the laser power was varied indicates that these fragments result from single-photon dissociation processes at both wavelengths. The slight increase in low-mass fragments suggests that if additional photons are absorbed, the primary products are internal fragments (including immonium ions), not sequence ions. The former require the cleavage of two backbone bonds while the latter can be generated after one such bond is broken. Therefore, when all other experimental parameters are the same, differences in the distribution of sequence ions in fragmentation spectra must result from either the photon energy or the electronic state excited by each wavelength, not the number of photons absorbed.

We will first compare the results obtained from the two photodissociation wavelengths in our tandem-TOF instrument, followed by the linear ion trap. As with our previous studies $[16,17]$, the spectra obtained with 157 $\mathrm{nm}$ photodissociation in our tandem-TOF instrument are dominated by ions resulting from cleavage of the peptide backbone between the $\alpha$ - and carbonyl-carbons. For peptides having arginine at their C-terminus, this yields a series of $x$ fragments with relatively consistent intensities. For Glu-fibrinopeptide B (Figure 1b), AAAANSAAAR (Figure 3b) and FSWGAEGQR (Figure $5 \mathrm{~b})$, complete $x$ series were observed. When arginine was located at the N-terminus, as with Substance P (Figure $7 b$ ), the complementary $a$ fragments were observed. $d, v$ or $w$ fragments corresponding to side-chain cleavage were also observed. Some low-energy fragments were observed in these experiments, and nearly 


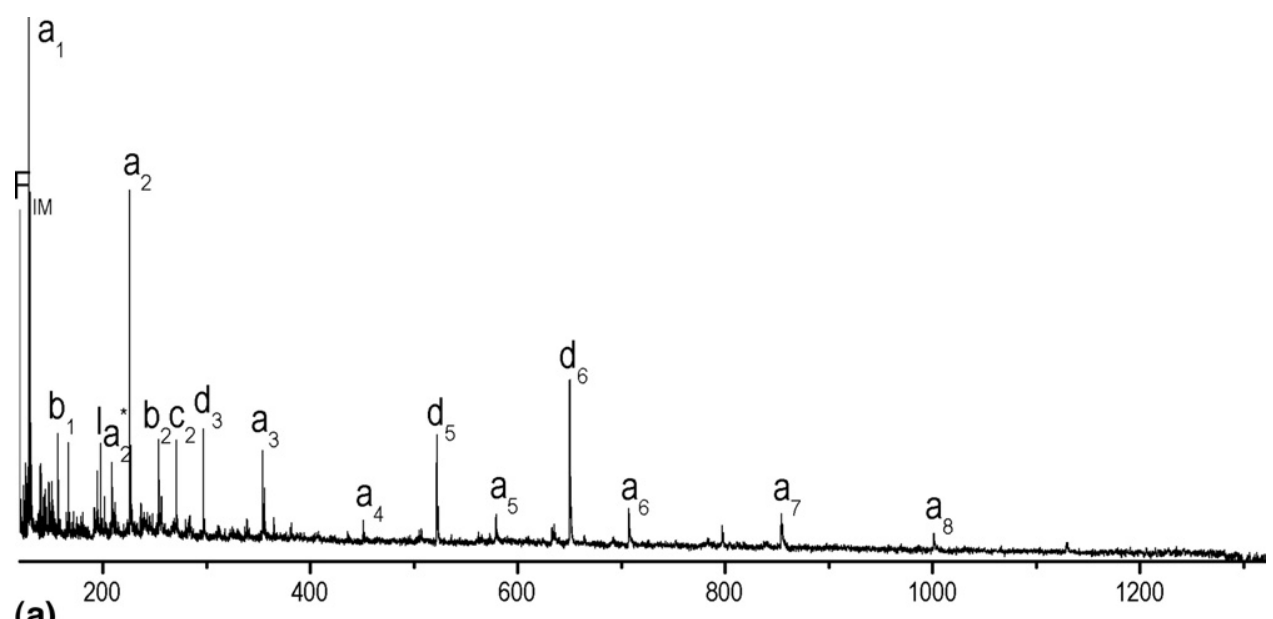

(a)

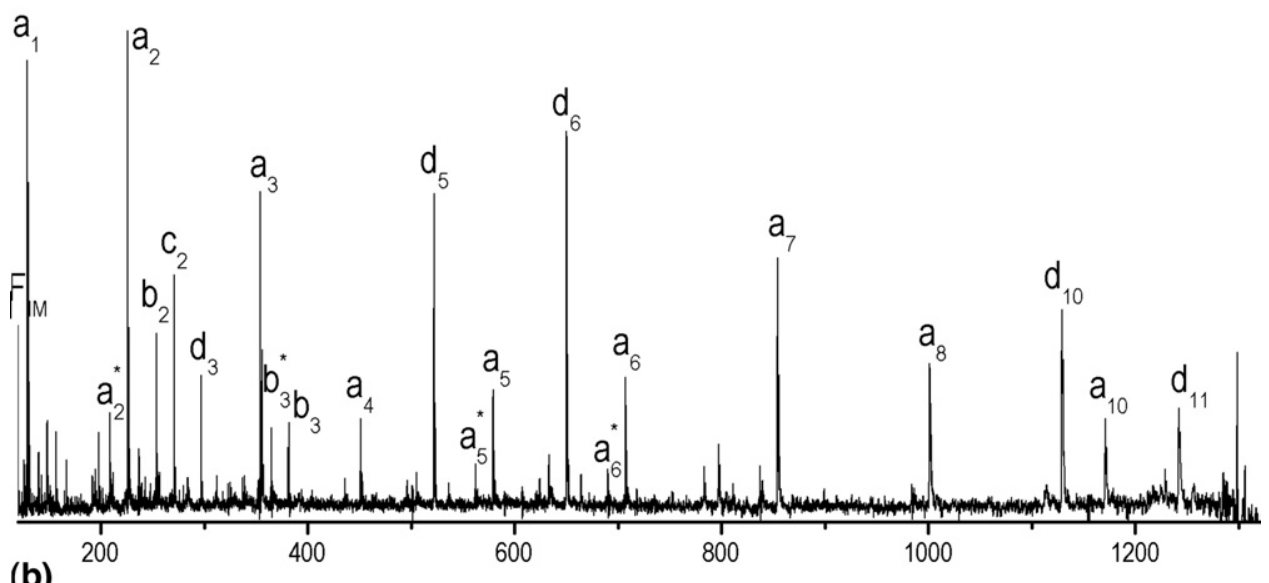

(b)

Figure 7. Tandem-TOF photodissociation of Substance P (RPKPQQFFGLM-NH ${ }_{2}$ ) using (a) 193 and (b) $157 \mathrm{~nm}$ light.

all were consistent with a charge being sequestered on arginine.

When the same experiments were performed using $193 \mathrm{~nm}$ light, the fragmentation spectra were significantly different. For Glu-fibrinopeptide B (Figure 1a), AAAANSAAAR (Figure 3a), and Substance P (Figure 7a) the largest fragment ion observed had about $70 \%$ of the precursor mass, with intensities decreasing with mass. While these spectra contain significant numbers of high-energy ions, their number and distribution are significantly different than with $157 \mathrm{~nm}$ light, as the high-mass fragments are missing. In contrast, no clear low mass bias is observed in the 193 tandem-TOF photodissociation of FSWGAEGQR (Figure 5a). Instead, an extensive collection of high-energy fragments is present, along with unusual peaks corresponding to the loss of the tryptophan and phenylalanine side chains from the intact peptide. These aromatic amino acids can absorb $193 \mathrm{~nm}$ light, and the unusual properties of this $193 \mathrm{~nm}$ photodissociation spectrum may be related to the presence of these additional chromophores. Further studies of peptides containing aromatic residues are warranted. Overall, the $193 \mathrm{~nm}$ spectrum of this peptide is quite similar to the $157 \mathrm{~nm}$ results from this instrument. From the tandem-TOF data alone, apparently differences in the photofragmentation process induced by the two wavelengths significantly influence the resulting fragmentation patterns.

As expected [20], most of the high-energy ions seen in $157 \mathrm{~nm}$ tandem-TOF photodissociation spectra were also observed when this wavelength was used in the linear ion trap. Glu-fibrinopeptide B (Figure 2b), AAAANSAAAR (Figure $4 \mathrm{~b}$ ), and FSWGAEGQR (Figure $6 \mathrm{~b}$ ) generated complete $x$ ion series. Surprisingly, the $193 \mathrm{~nm}$ photodissociation spectra for each peptide were quite similar to the $157 \mathrm{~nm}$ results from this instrument; near-complete $x$ ion series were observed. These were accompanied by a large number of lowenergy fragments. For example, seven $y$ ions appear in the $157 \mathrm{~nm}$ ion trap photodissociation of AAAANSAAAR (Figure $4 b$ ), while only three are observed with the tandem-TOF instrument (Figure $3 b$ ). In several cases, such as the previously described $y_{6}$ and $y_{9}$ peaks from Glu-fibrinopeptide B (Figure 2a and b) fragments corresponding to preferential cleavages adjacent to acidic amino acids were particularly intense in the ion 


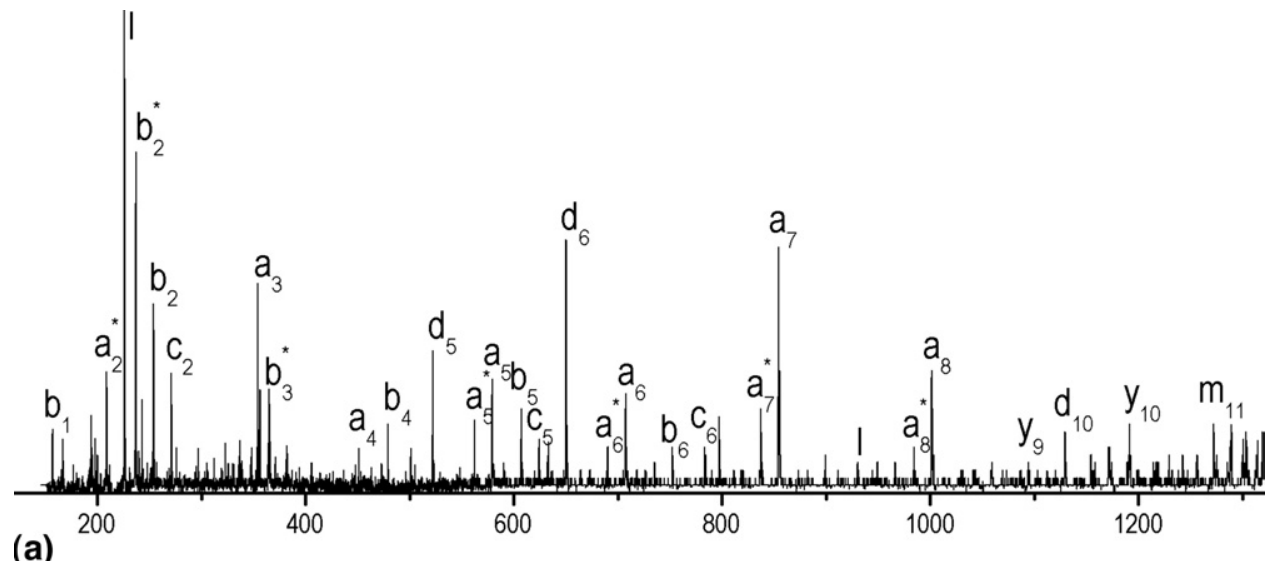

(a)

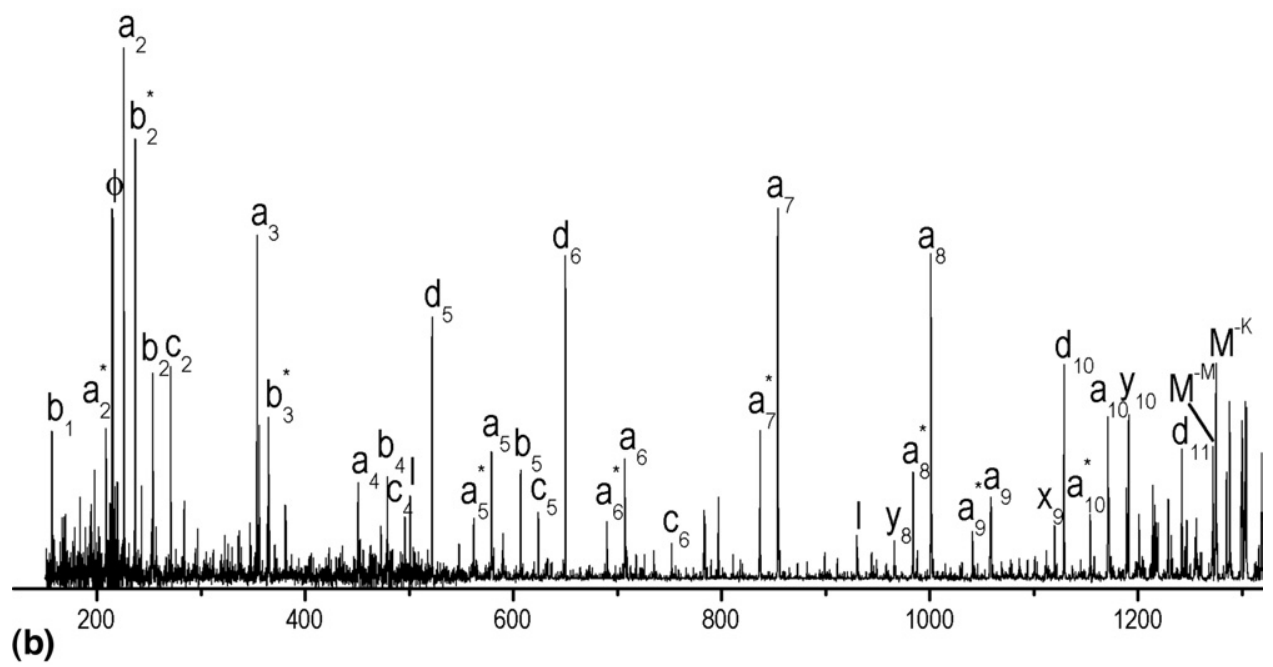

Figure 8. Linear ion trap photodissociation of Substance P (RPKPQQFFGLM-NH ${ }_{2}$ ) using (a) 193 and (b) $157 \mathrm{~nm}$ light.

trap. Similarly, while a $y_{3}$ peak was observed from FSWGAEGQR with both instruments, its relative intensity was significantly higher with both wavelengths in the trap. More fragments corresponding to chargetransfer away from arginine are present in ion trap than tandem-TOF spectra. These include N-terminal $a$ and $b$ ions from AAAANSAAAR (Figure $4 b$ ),$b$ ions from FSWGAEGQR (Figure 6b), and C-terminal fragments from Substance $\mathrm{P}$ (Figure $8 \mathrm{~b}$ ). In contrast with the conclusion from the tandem-TOF results, the similarity of the 157 and $193 \mathrm{~nm}$ ion trap spectra suggests that both wavelengths can induce similar fragmentation processes.

Two significant differences between the tandem-TOF and ion trap instruments could influence the photodissociation spectra that they produce. One is the observation timescale, as has been previously suggested [19, 20]. In our tandem-TOF instrument, the time between photoexcitation and subsequent ion extraction into the second mass analyzer is about $1 \mu \mathrm{s}$, while in the linear ion trap, the equivalent time is greater than $10 \mathrm{~ms}$. A second significant difference involves buffer gas. In the trap, collisions ensure that ions have near-thermal en- ergies before photodissociation and they provide cooling after light absorption. In contrast, collision probabilities in the tandem-TOF are nearly zero, and all internal energy imparted during the MALDI and photoexcitation processes is retained.

Two groups have performed VUV peptide photodissociation experiments using TOF instruments with observation timescales intermediate between those of our two mass analyzers. Since only $193 \mathrm{~nm}$ photodissociation data are available from all instruments, we will first compare photodissociation at this wavelength, and then use this information to aid in interpretation of all photodissociation spectra.

Barbacci and Russell have used two types of TOF instrument to perform $193 \mathrm{~nm}$ peptide photodissociation studies. In their original stepped-reflectron spectrometer, the time between photoexcitation and fragment ion separation was about $10 \mu \mathrm{s}$ [12]. More recently, Morgan and Russell have performed experiments on some of the same peptides using a tandemTOF instrument [19]. The critical time between photoexcitation and ion re-acceleration in this instrument, about $1 \mu \mathrm{s}$, is similar to that in our tandem-TOF. They 


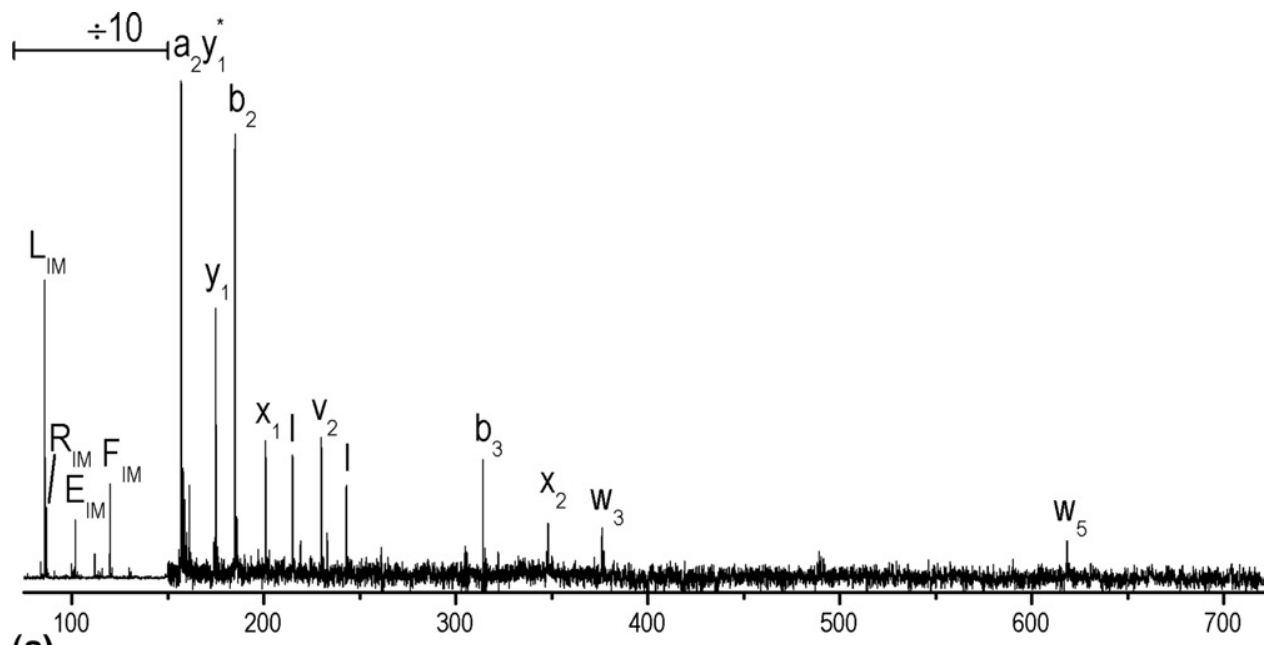

(a)

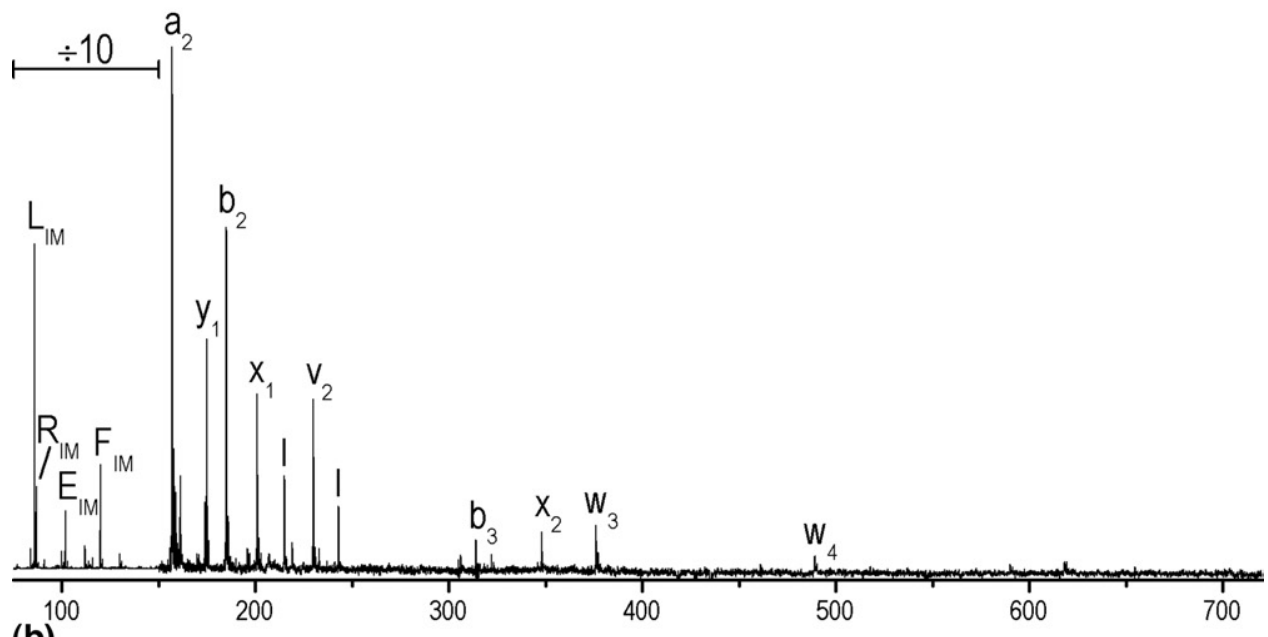

(b)

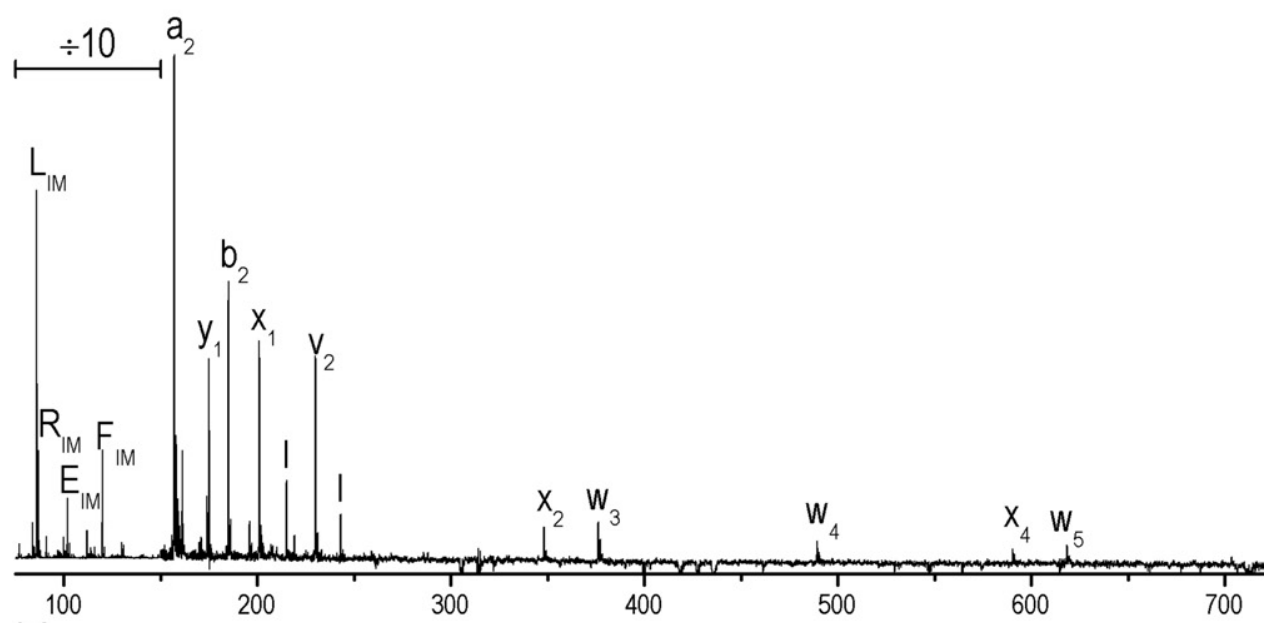

(c)

Figure 9. $193 \mathrm{~nm}$ Tandem-TOF photodissociation of ALELFR using (a) $1 \mathrm{~mJ}$, (b) $4.3 \mathrm{~mJ}$, and (c) 7.3 $\mathrm{mJ}$ light. Precursor ion depletion rates were (a) $41 \%$, (b) $68 \%$, and (c) $80 \%$

observed fewer $b$ and $y$ fragments with their tandem$\mathrm{TOF}$, and attributed this difference to the shorter time available for ion fragmentation [19]. As described above, both 157 and $193 \mathrm{~nm}$ photodissociation in the linear ion trap yield fragmentation spectra containing numerous low- and high-energy fragments with relatively little mass dependence. The increased number of low-energy fragments relative to the tandem-TOF re- 

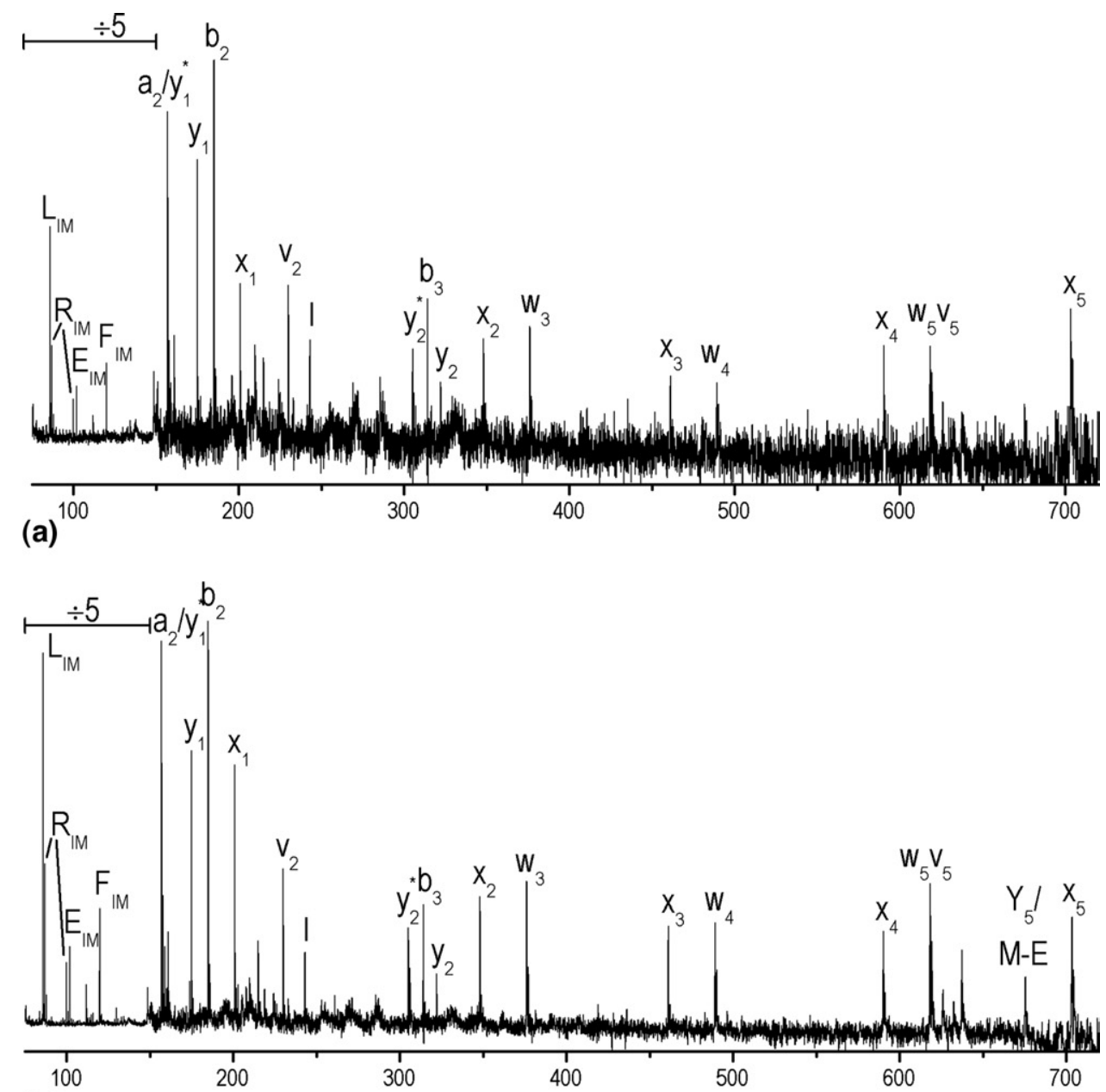

(b)

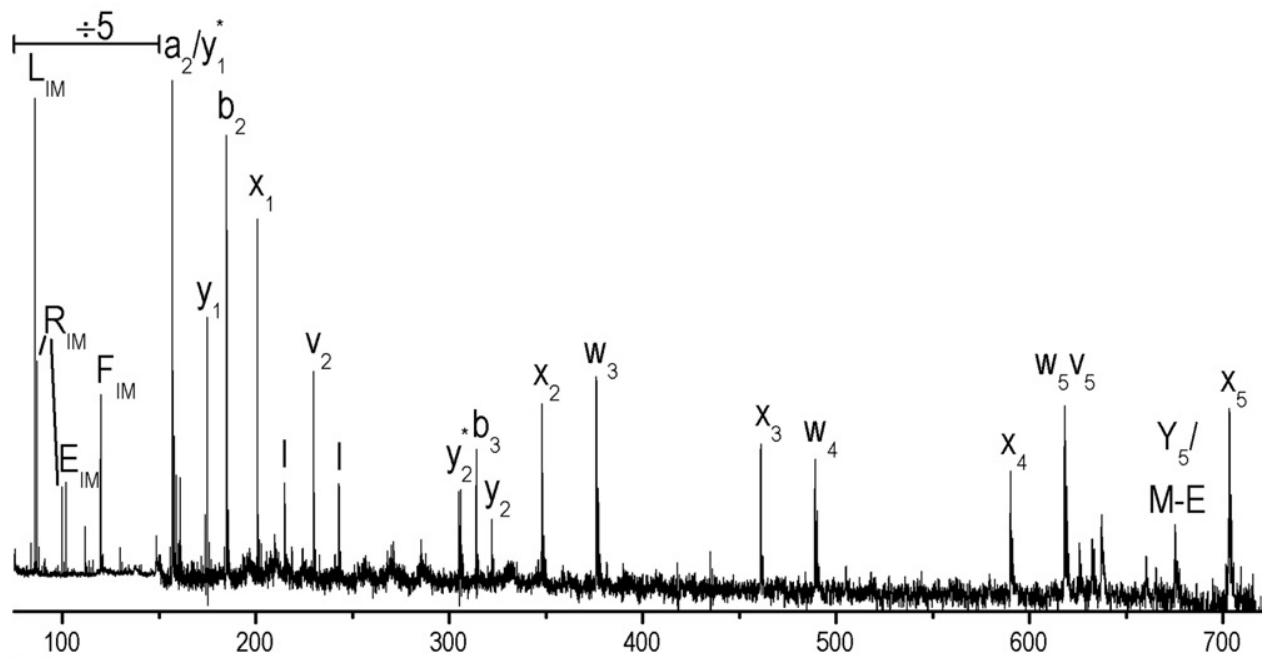

(c)

Figure 10. $157 \mathrm{~nm}$ Tandem-TOF photodissociation of ALELFR using (a) $1.2 \mathrm{~mJ}$, (b) $3 \mathrm{~mJ}$, and (c) 4.8 mJ light. Precursor ion depletion rates were (a) $8.4 \%$, (b) $24 \%$, and (c) $53 \%$.

sults is consistent with Morgan and Russell's observation of additional $b$ and $y$ ions when longer fragmentation times are available in $193 \mathrm{~nm}$ photodissociation experiments.
The collections of $x$ or $a$ fragments generated at 193 $\mathrm{nm}$ in the trap are surprisingly extensive compared with the limited number of these fragments typically observed at this wavelength in our tandem-TOF. The 
Table 1. Summary of fragment types typically observed in each experiment for peptides having C-terminal arginine

\begin{tabular}{|c|c|c|c|c|c|c|c|}
\hline \multirow[b]{2}{*}{ Mass analyzer } & \multirow[b]{2}{*}{$\begin{array}{c}\text { Observation } \\
\text { timescale }\end{array}$} & \multicolumn{3}{|c|}{$193 \mathrm{~nm}$ photofragments } & \multicolumn{3}{|c|}{$157 \mathrm{~nm}$ photofragments } \\
\hline & & $x$-type & $y$-type & $\begin{array}{l}\text { Acidic } \\
\text { residue } y\end{array}$ & $x$-type & $y$-type & $\begin{array}{l}\text { Acidic } \\
\text { residue } y\end{array}$ \\
\hline Tandem-TOF & $1 \mu \mathrm{s}$ & $\begin{array}{l}\text { Mass- } \\
\text { dependent }\end{array}$ & yes & no & Complete & yes & no \\
\hline Curved field TOF & $10 \mu \mathrm{s}$ & Complete & yes & no & $\mathrm{n} / \mathrm{a}$ & $\mathrm{n} / \mathrm{a}$ & $\mathrm{n} / \mathrm{a}$ \\
\hline Linear lon trap & $10 \mathrm{~ms}$ & Complete & yes & yes & Complete & yes & yes \\
\hline
\end{tabular}

peptides used by Russell's group did not have terminal arginine residues, making comparison of these highenergy fragments difficult. In the $193 \mathrm{~nm}$ curved-field reflectron TOF photodissociation studies of Kim and coworkers, the time between photoexcitation and fragment separation was about $10 \mu \mathrm{s}$ [24], similar to Russell's first apparatus. Since they analyzed three of the peptides used in the present study [25], we will directly compare their spectra with $193 \mathrm{~nm}$ results from our two instruments. Kim and coworkers observed a complete $x$ ion series along with strong $w_{8}$ and $w_{9}$ fragments and 8 $y$-type fragments for Glu-fibrinopeptide B (EGVNDNEEGFFSAR) [25]. Fragment ions $y_{6}$ and $y_{9}$ are particularly intense in our ion trap spectrum, yet they do not appear in the curved-field reflectron data. Kim and coworkers also observed analogous behavior for the peptide FSWGAEGQR, with a near-complete $x$ ion series. While the $y_{3}$ peak in our linear ion trap data for this molecule is intense, it is significantly weaker in Kim's curved-field TOF experiments. As described above, these $y$-type ions are the result of a specific fragmentation process associated with the side chains of acidic amino acids. Photodissociation of one other peptide, Substance P, has also been reported on all three instruments. The curved field reflectron photofragmentation spectrum is very similar to our $193 \mathrm{~nm}$ ion trap spectrum (Figure 8a). Most noteworthy, Kim and coworkers observe more high-energy fragments than we do with our tandem-TOF at this wavelength. In fact, their $193 \mathrm{~nm}$ spectra exhibit significant similarity with our $157 \mathrm{~nm}$ tandem-TOF results, with similar distributions of both low- and high-energy fragments.

The differences between $193 \mathrm{~nm}$ photodissociation spectra acquired using the three instruments suggest that the timescale available for ion fragmentation is critical. For reasons that are not clear, the short observation timescale of the tandem-TOF instrument appears to reduce the signal from high mass high-energy fragments. When this time increases by one order of magnitude in the curved-field reflectron instrument, a nearly complete high-energy fragment series is observed. Although the timescale of the linear ion trap is far longer, and buffer gas is also present, the only clear difference between ion trap and curved-field reflectron data is an increase in the abundance of ions resulting from charge-remote preferential cleavages. Since these fragments result from the rearrangement of acid amino acid side chains, they may form more slowly than other $y$-type fragments. The overall similarity between 193 $\mathrm{nm}$ curved-field reflectron and linear ion trap results suggests that ion cooling by the buffer gas does not have a dramatic influence on the observed photofragment distributions.

Our observation of peptide backbone cleavage adjacent to vacuum ultraviolet chromophores in $157 \mathrm{~nm}$ photodissociation tandem-TOF experiments led us to suggest that this phenomenon involves a prompt photochemical process [16]. However, the large collection of additional fragment types produced in $157 \mathrm{~nm}$ ion trap experiments indicates that other processes are occurring. In particular, the observation of low-energy $y$-type fragments suggests that at some point energy randomization takes place. This may occur before any bond is cleaved, either as an alternative pathway after excitation of the backbone amide, or due to absorption by a different chromophore such the amino acid side chains described above. Alternatively, the internal energy imparted by a photon at either wavelength could be sufficient to cause fragmentation through nonphotochemical processes such as shattering [34]. Since the energy imparted by absorption of a 193 or $157 \mathrm{~nm}$ photon is higher than the energy of a peptide bond, primary ions formed by photodissociation will still have significant internal energy. This energy could generate low-energy fragment ions by secondary processes. The differences observed between instruments suggest that the fragmentation times for these processes can vary significantly. Depending on the observation timescale and excitation wavelength, similar fragments could be formed by different mechanisms. From the available data it is impossible to determine the roles that these processes play in various photofragmentation experiments.

\section{Conclusions}

The present results demonstrate that photofragmentation of peptide ions by vacuum ultraviolet light does not generate a single characteristic, prototypical spectrum. In fact, observed ion distributions depend on the peptide sequence, the light wavelength, and the mass analyzer that is employed. Certain amino acids may influence photodissociation patterns by providing alternative chromophores that affect the absorption of light and the subsequent photochemistry. The photodissociation wavelength determines both the energy imparted 
to the analyte and the accessible photochemical pathways. Mass analyzers are sensitive to different fragmentation timescales and this can limit the dissociation processes that are observable. Discrepancies among reported peptide photofragmentation results can now be rationalized based on consideration of these factors. The particular combination of mass analyzer and photodissociation wavelength that generates the most readily interpretable information, particularly in proteomic applications, remains to be determined.

\section{Acknowledgments}

This work has been supported by NSF grants CHE0518234 and CHE0431991. It was also supported by the Indiana METACyt Initiative of Indiana University, funded in part through a major grant from the Lilly Endowment, Inc.

\section{References}

1. Robin, M. Higher Excited States of Polyatomic Molecules, Vol. II; Academic Press: Orlando, FL, 1975, p. 139.

2. Peterson, D. L.; Simpson, W. T. Polarized Electronic Absorption Spectrum of Amides with Assignments of Transitions. J. Am. Chem. Soc. 1957, 79, 2375-2382.

3. Clark, L. B. Polarization Assignments in the Vacuum UV Spectra of the Primary Amide, Carboxyl, and Peptide Groups. J. Am. Chem. Soc. 1995, 117, 7974-7986

4. Woody, R. W.; Koslowski, A. Recent Developments in the Electronic Spectroscopy of Amides and $\alpha$-Helical Polypeptides. Biophys. Chem. 2002, 101/102, 535-551.

5. Sleno, L.; Volmer, D.; A. Ion Activation Methods for Tandem Mass Spectrometry. J. Mass Spectrom. 2004, 39, 1091-1112.

6. Bowers, W. D.; Delbert, S. S.; Hunter, R. L.; McIver, R. T., Jr. Fragmentation of Oligopeptide Ions Using Ultraviolet Laser Radiation and Fourier Transform Mass Spectrometry. J. Am. Chem. Soc. 1984, 106, 7288-7289.

7. Hunt, D. F.; Shabanowitz, J.; Yates, J. R. III. Peptide Sequence Analysis by Laser Photodissociation Fourier Transform Mass Spectrometry. J. Chem. Soc. Chem. Commun. 1987, 8, 548-550.

8. Williams, E. R.; Furlong, J. J. P.; McLafferty, F. W. Efficiency of Collisionally-Activated Dissociation and 193-nm Photodissociation of Peptide Ions in Fourier Transform Mass Spectrometry. J. Am. Soc. Mass Spectrom. 1990, 1, 288-294.

9. Williams, E. R.; Henry, K. D.; McLafferty, F. W.; Shabanowitz, J.; Hunt, D. F. Surface-Induced Dissociation of Peptide Ions in Fourier-Transform Mass Spectrometry. J. Am. Soc. Mass Spectrom. 1990, 1, 413-416.

10. Martin, S. A.; Hill, J. A.; Kittrell, C.; Biemann, K. Photon-Induced Dissociation with a Four-Sector Tandem Mass Spectrometer. J. Am. Soc. Mass Spectrom. 1990, 1, 107-109.

11. Harris, W. A.; Reilly, J. P. Photodissociation in a Two-Stage Time-ofFlight Mass Spectrometer. Proceedings of the 46th ASMS Conference on Mass Spectrometry; Orlando, FL, 1998.

12. Barbacci, D. C.; Russell, D. H. Sequence and Side-Chain Specific Photofragment (193 nm) Ions from Protonated Substance P by MatrixAssisted Laser Desorption Ionization Time-of-Flight Mass Spectrometry. J. Am. Soc. Mass Spectrom. 1999, 10, 1038-1040.

13. Hettick, J. M.; McCurdy, K. L.; Barbacci, D. C.; Russell, D. H. Optimization of Sample Preparation for Peptide Sequencing by MALDI-TOF Photofragment Mass Spectrometry. Anal. Chem. 2001, 73, 5378-5386.
14. Thompson, M. S.; Cui, W.; Reilly, J. P. MALDI Photodissociation TOF-TOF Mass Spectrometry. Proceedings of the 51st ASMS Conference on Mass Spectrometry; Montreal, Canada, June 2003.

15. Harris, W. A.; Reilly, J. P. Photodissociation of small proteins in a two-stage linear time-of-flight mass spectrometer. Proceedings of the 45 th ASMS Conference on Mass Spectrometry; Palm Springs, CA, June 1997.

16. Thompson, M. S.; Cui, W.; Reilly, J. P.Fragmentation of Singly-Charged Peptides by Photodissociation at $\lambda=157 \mathrm{~nm}$. Angew. Chem. Int. Ed. 43, 4791, 2004, 4794.

17. Cui, W.; Thompson, M. S.; Reilly, J. P. Pathways of Peptide ion Fragmentation Induced by Vacuum Ultraviolet Light. J. Am. Soc. Mass Spectrom. 2005, 16, 1384-1398.

18. Papayannopoulos, I. A. The Interpretation of Collision-Induced Dissociation Tandem Mass Spectra of Peptides. Mass Spectrom. Rev. 1995, 14 , 49-73.

19. Morgan, J. W.; Russell, D. H. Comparative Studies of 193-nm Photodissociation and TOF-TOFMS Analysis of Bradykinin Analogues: The Effects of Charge Site(s) and Fragmentation Timescales. J. Am. Soc. Mass Spectrom. 2006, 17, 721-729.

20. Kim, T. Y.; Thompson, M. S.; Reilly, J. P. Peptide Photodissociation at $157 \mathrm{~nm}$ in a Linear Ion Trap Mass Spectrometer. Rapid Commun. Mass Spectrom. 2005, 19, 1657-1665.

21. Fung, Y. M. E.; Kjeldsen, F.; Silivra, O. A.; Chan, T. W. D.; Zubarev, R. A. Facile Disulfide Bond Cleavage in Gaseous Peptide and Protein Cations by Ultraviolet Photodissociation at $157 \mathrm{~nm}$. Angew. Chem. Int. Ed. 44, $6399,2005,6403$

22. Kjeldsen, F.; Savitski, M. M.; Adams, C. M.; Zubarev, R. A. Determination of the Location of Positive Charges in Gas-Phase Polypeptide Polycations by Tandem Mass Spectrometry. Int. J. Mass Spectrom. 2006, $252,204-212$

23. Kjeldsen, F.; Silivra, O. A.; Zubarev, R. A.Zwitterionic States in GasPhase Polypeptide Ions Revealed by $157 \mathrm{~nm}$ Ultraviolet Photodissociation. Chem. Eur. J. 12, 7920, 2006, 7928.

24. Moon, J. H.; Yoon, S. H.; Kim, M. S.Construction of an Improved Tandem Time-of-flight Mass Spectrometer for Photodissociation of Ions Generated by Matrix-Assisted Laser Desorption Ionization (MALDI). Bull. Korean Chem. Soc. 26, 763, 2005, 768 .

25. Moon, J. H.; Yoon, S. H.; Kim, M. S. Photodissociation of Singly Protonated Peptides at $193 \mathrm{~nm}$ Investigated with Tandem Time-ofFlight Mass Spectrometry. Rapid Commun. Mass Spectrom. 2005, 19, 3248-3252.

26. Choi, K. M.; Yoon, S. H.; Sun, M.; Oh, J. Y.; Moon, J. H.; Kim, M. S. Characteristics of Photodissociation at $193 \mathrm{~nm}$ of Singly Protonated Peptides Generated by Matrix-Assisted Laser Desorption Ionization (MALDI). J. Am. Soc. Mass Spectrom. 2006, 17, 1643-1653.

27. Moon, J. H.; Shin, Y. S.; Cha, H. J.; Kim, M. S. Photodissociation at 193 $\mathrm{nm}$ of Some Singly Protonated Peptides and Proteins with $\mathrm{m} / \mathrm{z} 2000$ 9000 Using a Tandem Time-of-Flight Mass Spectrometer Equipped with a Second Source for Delayed Extraction/Post-Acceleration of Product Ions. Rapid Commun. Mass Spectrom. 2007, 21, 359-368.

28. Suckau, D.; Resemann, A.; Schuerenberg, M.; Hufnagel, P.; Franzen, J. Holle, A. A novel MALDI LIFT-TOF/TOF Mass Spectrometer for Proteomics. Anal. Bioanal. Chem. 2003, 376, 952-965.

29. Medzihradszky, K. F.; Campbell, J. M.; Baldwin, M. A.; Falick, A. M. Juhasz, P.; Vestal, M. L.; Burlingame, A. L. The Characteristics of Peptide Collision-Induced Dissociation Using a High-Performance MALDI-TOF/TOF Tandem Mass Spectrometer. Anal. Chem. 2000, 72 552-558.

30. Wysocki, V. H.; Tsaprailis, G.; Smith, L. L.; Breci, L. A. Mobile and Localized Protons: A Framework for Understanding Peptide Dissociation. J. Mass Spectrom. 2000, 35, 1399-1406.

31. Biemann, K. Nomenclature for Peptide Fragment Ions (Positive-Ions). Methods Enzymol. 1990, 193, 886-887.

32. Gimon-Kinsel, M. E.; Kinsel, G. R.; Edmondson, R. D.; Russell, D. H. Photodissociation of High Molecular Weight Peptides and Proteins in a Two-Stage Linear Time-of-Flight Mass Spectrometer. J. Am. Soc. Mass Spectrom. 1994, 6, 578-587.

33. Reilly, J. P.; Kompa, K. L. Laser-Induced Multi-Photon Ionization Mass-Spectrum of Benzene. J. Chem. Phys. 1980, 73, 5468-5476.

34. Laskin, J.; Bailey, T. H.; Futrell, J. H. Shattering of Peptide Ions on Self-Assembled Monolayer Surfaces. J. Am. Chem. Soc. 2003, 125, 16251632. 